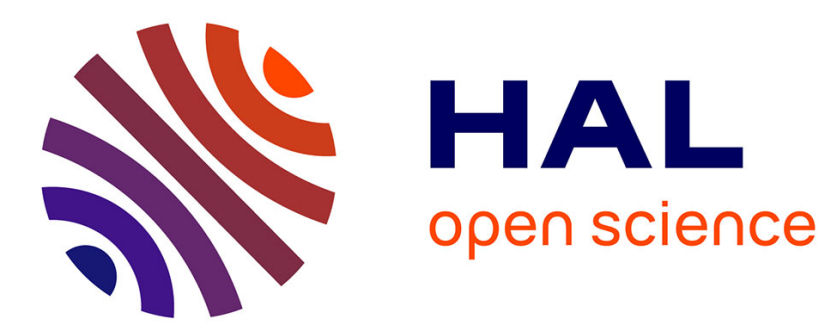

\title{
SUR LA RECHERCHE DU BACTERIUM COLI DANS LE LAIT (Suite)
}

Jean Pien, Jacques Bachimont

\section{To cite this version:}

Jean Pien, Jacques Bachimont. SUR LA RECHERCHE DU BACTERIUM COLI DANS LE LAIT (Suite). Le Lait, 1932, 12 (120), pp.1058-1071. hal-00895085

\section{HAL Id: hal-00895085 \\ https://hal.science/hal-00895085}

Submitted on 1 Jan 1932

HAL is a multi-disciplinary open access archive for the deposit and dissemination of scientific research documents, whether they are published or not. The documents may come from teaching and research institutions in France or abroad, or from public or private research centers.
L'archive ouverte pluridisciplinaire HAL, est destinée au dépôt et à la diffusion de documents scientifiques de niveau recherche, publiés ou non, émanant des établissements d'enseignement et de recherche français ou étrangers, des laboratoires publics ou privés. 
comme très souvent lorsqu'ils proviennent d'un intestin où l'on trouve du B. d'Eberth ou du Para B.

Les mandataires aux Halles ont remarqué depuis longtemps que les beurres centrifugés de certaines laiteries ne se conservaient pas. Cependant, ees établissements ont fait quelquefois des dépenses considérables pour s'approvisionner en eau par forages profonds. Malheureusement, la profondeur ne signifie rien si le terrain est calcaire et perméable avec, aux environs, des absorptions d'eaux usées.

En communiquant ces résultats, j'attire tout particulièrement l'attention sur la mauvaise qualité bactériologique de certains beurres et j'émets le vœu qu'une étude sur place puisse permettre d'envisager les moyens d'améliorer celle-ci. Il y a là une cause nouvelle d'étiologie de la fièvre typhoïde qu'il est indispensable de supprimer, parce que la consommation du beurre cru est très répandue.

\title{
SUR LA RECHERCHE DU BACTERIUM COLI DANS LE LAIT
}

\author{
JEAN PIEN \\ Ingénieur chimiste (I. C. R.) \\ Docteur ès Sciences \\ Directeur des Laboratoires \\ des "Fermiers Réunis "
}

par
et JACQUES BACHIMONT
du Laboratoire de Bactériologie des "Fermiers Réunis"

\section{(Suite).}

\section{DEUXIÈME ARTICLE.}

Dans notre précédent article sur cette question nous croyons avoir montré l'intérêt qui s'attache à la recherche du $B$. Coli dans le lait (raisons d'ordre hygiénique et d'ordre industriel). Nous avons insisté également sur la nécessité, surtout dans le contrôle industriel, d'effectuer cette recherche sur des quantités de lait bien supérieures à $1 \mathrm{~cm}^{3}$, puisque, dans ce dernier eas, le résultat est limité à 1.000 germes au litre, alórs qu'il y a souvent intérêt (dans l'industrie surtout) à déceler de plus faibles quantités de colibacilles. C'est ainsi qu'on peut même être amené à rechercher si un lait contient moins de 50, moins de 20 germes de $B$. coli au litre. La nécessité d'opérer sur d'assez grands volumes de lait pour déceler de petites quantités de coli nous paraît évidente (1).

(1) L'ingénieuse méthode de Mac Crady [1], qui consiste à opérer sur de petite volumes à l'aide d'un certain nombre de tubes en séries parallèles de dilutions différentes et à interpréter les résultats en s'aidant d'une formule mathématique, doit être à notre avis réservée aux teneurs élevées en colibacilles et ne semble pas applicable ici. 


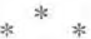

Plaçons-nous donc dans le cas le plus défavorable et supposons que nous soyons en présence d'un échantillon ou d'un prélèvement de lait d'un volume important (50 ou $100 \mathrm{~cm}^{3}$ ) et que, dans ce grand volume, il n'y ait que quelques germes de $B$. Coli ou même un seul (1).

Comment mettrons-nous leur présence en évidence?

La condition essentielle à remplir est, incontestablement, de faire proliférer aussi abondamment que possible le colibacille. Et la première idée qui se présente à l'esprit pour atteindre ce but est l'incubation pure et simple du lait à l'étuve.

Cette incubation devrait théoriquement être telle qu'on puisse ensuite :

$1^{\circ}$ Soit produire dans le lait lui-même des substances aisément identifiables ;

$2^{\circ}$ Soit (au cas où l'hypothèse précédente serait irréalisable) repiquer après prolifération suffisante, un petit volume de lait dans d'autres milieux de culture aux fins d'identification.

Nous démontrerons, ci-après, que la première hypothèse ne peut pas être retenue. La seconde devra donc être étudiée; elle pose comme condition essentielle que la prolifération du coli soit telle que le repiquage subséquent emporte certainement, dans tous les cas, des germes de B. Coli.

Fixons les idées sur un exemple pratique pris dans un cas extrême :

Soit un échantillon de $100 \mathrm{~cm}^{3}$ renfermant 1 germe de $B$. Coli (soit 10 au litre); il faut que l'incubation ait donné naissance théoriquement à 100 germes (pratiquement 500 ou 1.000, en raison de l'hétérogénéité de la répartition des germes) pour avoir la certitude, absolument nécessaire, de prélever au moins 1 germe en repiquant $1 \mathrm{~cm}^{3}$ de lait sur un autre milieu après l'incubation. Faute de quoi on conclurait à l'absence de coli même quand il y en a beaucoup plus de 10 au litre, ainsi que nous le démontrerons. Il est donc indispensable, pour poursuivre l'étude de cette seconde hypothèse, de connaître le mode de prolifération du coli en lait dans les conditions d'une incubation normale afin de savoir au bout de combien d'heures le nombre de germes de coli initiaux se sera multiplié par 100, 500, ou $1.000 \ldots$, comme cela est indispensable.

Connaissant les conditions à réaliser dans le cas théorique du lait pur ne contenant que du colibacille, nous serons amenés à nous demander ce qui se passe dans la pratique, c'est-à-dire sur du lait renfermant d'autres germes et en particulier des ferments lactiques.

(1) Répétons que cet ordre de grandeur existe dans la pratique industrielle; il y a lieu de l'envisager dans le contrôle de la pasteurisation, en raison de tout ce que nous avons dit dans l'article précédent. Il n'y a là aucune exagération, aucune fiction. 
Notre plan sera done le suivant :

Première partie : Etude de la prolifération du coli par incubation en lait.

10 Production dans le lait lui-même de produits d'identification spécifiques.

$2^{\circ}$ Obtention d'abord en lait pur, puis en présence de certains autres microbes d'une prolifération de coli suffisante pour avoir des repiquages positifs dans tous les cas.

Deuxième partie : Etude de l'isolement et de l'identification du colibacille.

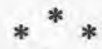

PREMIERE PARTIE.

\section{ÉTUDE DE LA PROLIFÉRATION DU COLI PAR INGUBATION EN LAIT.}

Le $B$. Coli se développe fort bien dans le lait tant que certaines conditions, fatales à son développement, ne sont pas atteintes du fait de la présence d'autres germes (ferments lactiques en particulier). Nous avons vérifié que, au début de l'incubation, la présence de ces germes étrangers à côté du coli ne l'empêche pas de donner naissance à ses produits normaux de désassimilation. On peut donc supposer qu'il serait facile de déceler dans une culture de coli, en présence d'autres microbes, certaines substances caractéristiques produites abondamment pendant les premières heures de l'incubation, même si ultérieurement le développement du coli doit être arrêté. Comme, d'autre part, nous avons vérifié que ces substances spécifiques subsistent après la mort du coli, même en présence d'un développement considérable des autres germes présents, il nous est venu tout naturellement à l'esprit l'idée suivante :

Peut-on déceler la présence du coli, même en petite quantité, dans un échantillon de lait par la caractérisation d'un produit de désassimilation spécifique obtenu au cours de l'incubation et conservé par la suite?

Malheureusement cette question, qui eût grandement simplifié le problème, doit recevoir une réponse négative.

\section{§ 1. - IMPOSSIBILITÉ DE PRODUIRE DANS LE LAIT LUI-MEME DES PRODUITS D'IDENTIFICATION SPECIFIQUES.}

\section{A. LE COLI NE DONNE PAS D'INDOL DANS LE LAIT.}

Le coli poussant dans le lait, donne principalement de l'acide lactique, de l'hydrogène, de l'acide carbonique et un peu de 
méthane. Aucune de ces substances n'est spécifique du coli et ne peut servir à l'identifier.

L'un des caractères les plus certains et les plus constants du $B$. Coli commune est la production de l'indol à partir des peptones. Et la réaction la meilleure pour déceler l'indol est la formation d'une coloration rouge par l'action de la paradiméthylaminobenzaldéhyde en milieu chlorhydrique (réaction d'Erlich-Kovacs). Cette réaction est très fidèle, suffisamment spécifique pour l'usage qui en est fait en bactériologie, et surtout d'une sensibilité très supérieure à tous les autres procédés de recherche de l'indol lui-même. Nous en ferons une étude détaillée dans la suite de ce travail.

La production de l'indol par elle-même n'est pas spécifique du colibacille (puisque le proteus vulgaris, le vibrion cholérique, par exemple, la donnent également); mais ces germes sont ou bien aisément séparables du colibacille par culture en présence d'antiseptiques, ou bien extrêmement rares dans le lait, pour ne pas dire toujours absents. De sorte qu'il n'y aurait pas, à priori, d'impossibilité théorique à caractériser le coli dans le lait, moyennant certains artifices de culture, par l'identification de l'indol si celui-ci pouvait se former dans le lait. Or l'indol est un produit de désintégration de la molécule de peptone que le lait ne renferme point normalement (1). Done, on pouvait prédire qu'il ne se formerait pas d'indol dans le lait - ee que l'expérience a pleinement confirmé.

Nous avons fait cultiver du coli en grande quantité dans du lait et nous avons, après 24 et 48 heures d'incubation à $37^{\circ}$, effectué sur le lait lui-même la réaction d'Erlich-Kovacs: nous n'avons obtenu de coloration rouge ni dans la caséine ni dans le sérum (2). La même quantité de coli dans les mêmes conditions de température et de durée en eau peptonée, a donné une coloration rouge vif dans l'alcool amylique surnageant le réactif d'ErlichKovacs.

Ce résultat, suffisant pour dire que "la réaction de l'indol est négative sur un lait où le coli a abondamment cultivé ", donc suffisant pour démontrer ce que nous cherchons pratiquement, nous autorise-t-il vraiment à dire que le coli ne donne pas d'indol dans le lait ?

Nous croyons intéressant d'ouvrir ici une parenthèse sur cette 'question.

D'après ce que nous savons des propriétés biochimiques du coli-

(1) D'après Hammarsten la présure et certains germes présurigènes donnerajent dans le lait certaines peptones.

(2) A la longue et suivant les conditions de température de la réaction, il peut se former une légère coloration dans la masse, même sur du lait stérile pur. Cette coloration est due à l'action de l'acide chlorhydrique du réactif sur la caséine du lait. 
bacille, il semble bien que ce microbe soit incapable de s'attaquer à la caséine pour aboutir à l'indol. Le colibacille est capable d'aller du tryptophane à l'indol en passant vraisemblablement par les stades tryptophane, acide indolacétique, scatol, acide indolcarbonique, indol. Mais il est peu probable qu'il puisse passer de la caséine au tryptophane - bien que certains microbes, par contre, semblent passer assez facilement de la gélatine au tryptophane (certains liquéfiants, par exemple). Le coli, qui ne liquéfie pas la gélatine, doit peu s'attaquer à la caséine ; il préfère d'ailleurs de beaucoup l'azote minéral à l'azote organique des protéines élevées, ainsi que nous aurons probablement l'occasion de le montrer.

Rien n'autorisait done à dire que le coli pouvait donner de l'indol dans le lait. Mais en revanche l'expérience négative précédente ne nous permet pas d'affirmer qu'il n'en peut pas donner ; ear on peut dire : Si de l'indol s'est formé, ne s'est-il pas fixé sur la molécule protéique, échappant ainsi à la réaction ? S'il s'en est formé, d'autres constituants du lait (lactose, par exemple) ne gênent-ils pas la réaction d'Erlich-Kovacs?

Nous avons cru devoir élucider ces points pour pouvoir émettre une certitude.

\section{$1^{\circ}$ De l'indol introduit dans le lait, y est décelable} directement malgré sa fixation sur la caséine. - Dans plusieurs tubes de lait stérile nous avons introduit des doses croissantes d'indol du même ordre de grandeur que celles que l'on rencontre dans des cultures abondantes en peptone après 24 heures d'étuve. La réaction obtenue à l'Erlich-Kovacs est positive. Mais au lieu d'avoir une coloration rouge dans l'alcool amylique surnageant, c'est la caséine qui fixe la coloration : le filtrat est incolore ou à peine coloré quand il y a excès d'indol. Cette laque est partiellement soluble dans le chloroforme, insoluble dans l'alcool amylique.

Nous avons pu mettre en évidence que c'est bien l'indol luimême qui se fixe sur la caséine et non les produits de la réaction. En effet : du lait est additionné d'indol, caillé à l'acide lactique et filtré ; le filtrat ne donne rien à l'Erlich-Kovacs (après avoir vérifié que l'acide lactique n'exerce aucune influence sur la réaction finale). Le caillé recueilli se colore nettement en rose par trituration avec le réactif. Donc, dans le lait, l'indol apporté se fixe sur la caséine et rien ne s'oppose à la formation dans le caillé d'une coloration rose caractéristique de l'indol par action du réactif d'Erlich-Kovacs. Il faut reconnaître que la sensibilité de la réaction de l'indol est diminuée en présence de lait; mais aux doses où l'indol pourrait se former dans une culture abondante de 24 heures, et même moins, cette quantité n'échapperait pas à la réaction.

En résumé, s'il s'était formé de l'indol dans le lait sa fixation 
éventuelle sur la caséine (d'ailleurs démontrée comme réelle) n'eût pas empêché de le déceler, et la première objection tombe.

$2^{\circ}$ Les autres constituants du lait ne gênent pas la réaction de l'indol. - L'expérience précédente suffirait à le démontrer. Mais il est intéressant, pour ce qui suit, d'en faire une démonstration directe.

Des solutions d'indol chargées ou non de lactose (jusqu'à $10 \%$ ) donnent exactement la même réaction positive. Le glucose n'a également aucune influence. Il en est de même de l'acide lactique (ce caractère est important à retenir). L'expérience a été refaite sur de l'eau peptonée et a donné le même résultat. Donc, le lactose, dans un liquide chargé d'indol, n'empêche pas la réaction de l'indol de se produire par le réactif d'Erlich-Kovacs et ne la modifie en rien.

Par conséquent, ni la caséine ni le lactose ne gênent la réaction de l'indol quand il s'en trouve dans le lait. Et la réaction négative obtenue. après incubation du coli en lait prouve bien qu'il ne s'est pas formé d'indol.

\section{B. LE COLI NE DONNE PAS DIINDOL DANS LE LAIT PEPTONÉ.}

Puisque le coli ne donne pas d'indol dans le lait (d'abord par suite de l'absence de peptones dans le lait), il vient immédiatement à l'esprit de refaire l'expérience en chargeant le lait de peptone avant l'incubation. Or, après 24 et 48 heures d'incubation à $37^{\circ}$ en présence d'une dose massivè de coli dans un lait peptoné à $2 \%$ (comme l'eau peptonée) on n'obtient aucune réaction positive par l'Erlich-Kovacs. Cette expérience répétée un nombre considérable de fois n'a souffert aucune exception.

Ce résultat, déconcertant à priori, méritait une étude systématique, qui a été faite et dont voici la conclusion : le lactose s'oppose à la production de l'indol par le colibacille en présence de peptone. Ce phénomène se produit dans l'eau peptonée lactosée, aussi bien que dans le lait peptoné.

Voici l'expérience fondamentale :

$10100 \mathrm{~cm}^{3}$ d'eau peptonée (à $2 \%$ ) contenant $1 \mathrm{~cm}^{3}$ d'une suspension aqueuse de coli (1) et incubés 24 heures à $37^{\circ}$, donnent une réaction d'Erlich-Kovacs très vive.

$2^{\circ} 100 \mathrm{~cm}^{3}$ d'eau peptonée (à $2 \%$ ) lactosée (à $5 \%$ comme le lait) contenant la même dose de coli et soumis à la même incubation, donnent une réaction nulle, quelquefois très faible cependant.

(1) Il faut avoir soin de s'adresser à une suspension aqueuse de colz, car une culture pré cédente en eau peptonée amènerait avec elle une certaine dose d'indol qui pourrait fausser les essais suivants ou les rendre douteux. 
$3^{\circ} 100 \mathrm{~cm}^{3}$ de lait pur contenant la même dose de coli et soumis à la même incubation, donnent une réaction nulle.

$4^{\circ} 100 \mathrm{~cm}^{3}$ de lait contenant $2 \%$ de peptone, la même dose de coli et soumis à la même incubation, donnent une réaction nulle, quelquefois très faible avec de plus grosses doses de coli au départ.

Nous avons montré précédemment que le lactose, même jusqu'à $10 \%$, ne gêne pas la réaction de l'indol une fois celui-ci formé.

Donc, il est hors de doute que le lactose empêche le coli de donner de l'indol dans les milieux peptonés dans les conditions normales de culture.

Remarques : $1^{0}$ Toutes ces recherches ont été également effectuées à l'aide d'autres réactifs moins sensibles de l'indol (Salkowsky, par exemple) et ont donné les mêmes résultats.

$2^{\circ}$ Le glucose joue exactement le même rôle que le lactose dans les mêmes conditions. Il empêche la production, mais il ne gêne pas la réaction de l'indol.

\section{§. - OBTENTION D'UNE PROLIFÉRATION SUFFISANTE DU COLI EN LAIT.}

Puisque l'hypothèse précédente sur la recherche directe du coli par la détection de l'indol dans le lait peptoné ou non n'a pas survécu à l'expérience, il devient nécessaire de faire proliférer le colibacille qui peut se trouver dans le lait soumis à l'analyse pour pouvoir, ensuite, le repiquer et le cultiver en d'autres milieux aux fins d'identification. La condition sine qua non de la réussite de cette technique est, avons-nous dit, un minimum de prolifération suffisante. Les quelques germes initiaux des cas les plus difficiles doivent être, à la fin de l'incubation, en nombre tel qu'un repiquage de $1 \mathrm{~cm}^{3}$ par exemple, ou même moins, apporte certainement des germes. Ce souci pourrait paraître puéril, puisque la multiplication à l'étuve des germes comme le coli dans des milieux de culture comme le lait, est extrêmement rapide et remplit la condition exigée en quelques heures. Mais il y a une marge entre la théorie et la pratique. Et dans la pratique le coli n'est jamais seul. D'autres germes se développent qui peuvent gêner celui-là, soit directement, soit indirectement par leurs produits de sécrétion. Il serait done téméraire de prescrire l'incubation sans savoir ce qui se passe et principalement sans connaître l'influence capitale des ferments lactiques et de l'acide lactique sur le développement du coli au cours de l'incubation.

Ce problème se subdivise donc en quelques autres; il nous faut en effet, d'abord connaître :

$1^{\circ}$ Le mode de prolifération du coli, supposé seul, en lait. 
$2^{\circ}$ L'influence des ferments lactiques sur le développement du coli, en l'absence d'acide lactique.

$3^{\circ}$ L'influence de l'acide lactique sur le développement du coli.

I. Mode de prolifération de colibacille en lait. - A quelle vitesse le coli se multiplie-t-il dans le lait? Au bout de combien d'heures les germes se seront-ils multipliés par $10,100,1.000, \ldots$ ? Il ne faut pas, dans ce domaine, prétendre à une précision absolue, mais seulement rechercher des ordres de grandeur.

L'expériènce a été faite à plusieurs reprises et a donné des résultats suffisamment concordants. Nous partions d'une suspension dans l'eau peptonée d'une colonie de B. Coli, dont on appréciait la richesse approximative par numération directe sous le microscope. Des fioles de $100 \mathrm{~cm}^{3}$ de lait stérile étaient ensemencées et mises à l'étuve. Pour la numération, après $4,5 \ldots, 9$ heures d'étuve, nous diluions le lait de telle façon que les boîtes de Pétri ne continssent pas moins de 30 germes, ni plus de 500 , conditions requises pour une numération exacte [2]. Le milieu employé était la gélose d'Orla-Jensen ; la numération se faisait après 3 jours de séjour à 370 (ce délai est largement suffisant pour le coli, dont les premières colonies apparaissent en quelques heures). Tous ces essais étaient faits en double avec des dilutions différentes. On ne conservait pour la numération que les boîtes ni trop chargées, qui donnent des résultats trop faibles, ni trop pauvres, qui donnent des résultats irréguliers, dus à un trop grand coefficient de dilution.

Voici les résultats de trois expériences faites à plusieurs semaines de distance sur des souches de coli différentes. Les chiffres

TABLEAU I.

NOMBRE DE GERMES DE B. GOLI PAR GENTIMËTRE GUBE DE LAIT.

\begin{tabular}{|c|c|c|c|c|c|c|}
\hline & \multicolumn{2}{|c|}{ Première expérience } & \multicolumn{2}{|c|}{ Deuxième expérience } & \multirow{3}{*}{$\begin{array}{r}\begin{array}{r}\text { Troisième } \\
\text { expérience }\end{array} \\
800\end{array}$} & \multirow[t]{2}{*}{$\begin{array}{l}\text { Quatrième } \\
\text { expérience }\end{array}$} \\
\hline & (a) & (b) & $(a)$ & (b) & & \\
\hline Au temps initial.... & 3.100 & 5.000 & 10.000 & 14.000 & & 7.000 \\
\hline $\begin{array}{l}\text { Après } 4 \text { heures d'in- } \\
\text { cubation } \ldots \ldots \ldots\end{array}$ & 566.000 & 740.000 & 2.200 .000 & 2.635 .000 & 156.000 & 852.000 \\
\hline $\begin{array}{l}\text { Après } 6 \text { heures d'in- } \\
\text { cubation ........ }\end{array}$ & 13.220 .000 & 16.400 .000 & 47.100 .000 & 55.500 .000 & 4.620 .000 & 12.420 .000 \\
\hline $\begin{array}{l}\text { Après } 7 \text { heures d'in- } \\
\text { cubation ... . . . }\end{array}$ & 一 & $62,000.000$ & 130.250 .000 & 167.500 .000 & 10.400 .000 & 一 \\
\hline $\begin{array}{c}\text { Après } 8 \text { heures d'in- } \\
\text { cubation ....... }\end{array}$ & 88.900 .000 & 131.000 .000 & 399.500 .000 & 485.000 .000 & 21.632 .000 & 100.000 .000 \\
\hline $\begin{array}{l}\text { Après } 9 \text { heures d'in- } \\
\text { cubation ... . . . . }\end{array}$ & 188.000 .000 & 230.000 .000 & 450.000 .000 & 一 & $\longrightarrow$ & 301.000 .000 \\
\hline
\end{tabular}


indiquent le nombre de germes vivants de $B$. Coli par centimètre cube de lait. Les deux premières expériences ont été faites en double.

Pour pouvoir juger plus aisément de la façon dont s'est multiplié le $B$. Coli au cours de cette incubation, il faudrait pouvoir ramener tous ces chiffres à un seul germe au temps initial. On aurait ainsi le coefficient de multiplication à chaque moment. Il suffit pour cela de diviser le nombre de germes de chaque temps par le nombre de germes $d u$ temps initial. A priori cette opération peut paraître choquante. Il est facile pourtant de montrer qu'elle est parfaitement licite. En effet :

Le colibacille se multiplie par scissiparité. Les nombres de germes qui apparaissent à chaque génération constituent les termes d'une progression géométrique de raison 2 . Si dans un intervalle donné le premier terme est $a$ germes, le dernier terme $l$ germes, et le nombre de termes $n$, on a :

$$
l=\mathrm{aq}^{\mathrm{n}-1}
$$

C'est la valeur bien connue du dernier terme d'une progression géométrique.

Or ce qui nous intéresse ici, c'est la somme de ces termes, dans l'intervalle. Dans une progression géométrique ordinaire, cette somme est évidemment différente du dernier terme. Mais dans une suite de générations bactériennes, chaque terme s'annule en donnant le terme suivant (un germe en donne deux, mais le premier germe disparaît du fait qu'il donne naissance aux deux autres). La somme de ces générations successives est donc égale au dernier terme, le seul qui subsiste. Si nous considérons par exemple l'intervalle 3.100-566.000 de la première expérience, nous pouvons affirmer, évidemment, que le dernier terme 566.000 représente la totalité des éléments qui se sont succédé dans la progression. Du fait de la scissiparité, la somme des termes, c'est bien le dernier terme.

Un germe initial donne un dernier terme égal à $2^{\mathrm{n}-1}(\mathrm{n}=\mathrm{nom}-$ bre de termes ou générations, $\mathrm{n}-1=$ nombre de germinations).

$$
\text { Or } \quad l=\operatorname{aq}^{\mathrm{n}-1} \quad \text { ou } \quad \frac{l}{\mathrm{a}}=\mathrm{q}^{\mathrm{n}-1}=2^{\mathrm{n}-1}
$$

Done, un germe initiale donne, en fin d'intervalle, un dernier terme, ou somme d'éléments finaux, égal à $\frac{l}{\mathrm{a}}$, c'est-à-dire au quotient du dernier terme par le premier terme. L'opération est donc licite. Elle fournit les résultats suivants, qui expriment le nombre de germes issus à chaque numération d'un seul germe au temps initial. 
TABLEAU II.

PRoliferation du B. GOLI A PARTIR D'UN SEUL Germe.

\begin{tabular}{|c|c|c|c|c|c|c|}
\hline \multirow[t]{2}{*}{1} & \multicolumn{2}{|c|}{ Première expérience } & \multicolumn{2}{|c|}{ Deuxième expérience } & \multirow{2}{*}{$\begin{array}{l}\text { Troisième } \\
\text { expérience }\end{array}$} & \multirow{2}{*}{$\begin{array}{l}\text { Quatrième } \\
\text { expérience }\end{array}$} \\
\hline & $(a)$ & $(b)$ & (a) & $(b)$ & & \\
\hline Au temps initial $\ldots \ldots \ldots \ldots$ & 1 & 1 & 1 & 1 & 1 & 1 \\
\hline Après 4 heures d'incubation . & 183 & 148 & 220 & 188 & 195 & 121 \\
\hline Après 6 heures d'incubation. & 4.270 & 3.280 & 4.710 & 3.960 & 5.775 & 1.770 \\
\hline Après 7 heures d'incubation. & $\longrightarrow$ & 12.400 & 13.025 & 11.960 & 13.000 & 一 \\
\hline Après 8 heures d'incubation. & 28.700 & 26.000 & 39.950 & 34.600 & 27.040 & 14.300 \\
\hline Après 9 heures d'incubation . & 60.600 & 46.000 & 45.000 & - & 一 & 43.000 \\
\hline
\end{tabular}

Ce tableau nous permet d'affirmer que le colibacille (à $37^{\circ}$, dans le lait) se multiplie par 100 après 3 ou 4 heures, par 1.000 après 5 ou 6 heures, par 10.000 en 7 heures environ. Bien entendu, il ne faut cesser de considérer ces données comme très approximatives, permettant de donner seulement un ordre de grandeur. Il faut toutefois rappeler que ces essais ne constituent pas une expérience en triple, mais trois expériences différentes effectuées avec des points de départ différents, ce qui augmente l'intérêt des résultats.

Remarques : $1^{\circ}$ Le tableau précédent permet encore des déductions curieuses en ce qui concerne la rapidité avec laquelle le coli se subdivise.

On peut en effet calculer le nombre de "germinations " ayant eu lieu dans chaque intervalle. S'il y a eu $n$ germinations à partir d'un germe, le dernier terme $l$ vaut $2^{\mathrm{n}}$; connaissant $l$ on déduit aisément $n$ à l'aide d'une table de logarithmes. (Il faut évidemment ramener à l'unité le nombre de germes du début de chaque intervalle.) Voici les valeurs de $n$ (nombre de germinations), dans chaque intervalle :

TABLEAU III.

NOMBRE DE GERMINATIONS DANS CHAQUE INTERVALLE

\begin{tabular}{|c|c|c|c|c|c|c|}
\hline & \multicolumn{2}{|c|}{ Première expérience } & \multicolumn{2}{|c|}{ Deuxième expérience } & \multirow{2}{*}{$\begin{array}{l}\text { Troisième } \\
\text { expérience }\end{array}$} & \multirow{2}{*}{$\begin{array}{l}\text { Quatrième } \\
\text { expérience }\end{array}$} \\
\hline & $(a)$ & (b) & (a) & $(b)$ & & \\
\hline De 0 à 4 heures d'incubation .... & 7,54 & 7,23 & 7,81 & 7,58 & 7,63 & 6,94 \\
\hline De 4 à 6 heures d'incubation .... & 4,56 & 4,48 & 4,43 & 4,41 & 4,90 & 3,90 \\
\hline De 6 à 7 heures d'incubation .... & & 1,92 & 1,46 & 1,60 & 1,18 & 302 \\
\hline De 7 à 8 heures d'incubation .... & 2,75 & 1,08 & 1,51 & 1,53 & 1,06 & 3,02 \\
\hline De 8 à 9 heures d'incubation .... & 1,08 & 0,82 & $0,16(?)$ & 一 & $\longrightarrow$ & 1,59 \\
\hline $\begin{array}{l}\text { Nombre total de germinations } \\
\text { en } 9 \text { heures } \ldots \ldots \ldots \ldots \ldots \ldots \ldots\end{array}$ & 15,93 & 15,53 & 15,43 & 15,12 & 14,77 & 15,45 \\
\hline
\end{tabular}


On en déduit le temps nécessaire à une "germination" dans chaque intervalle :

TABLEAU IV.

TEMPS NÉGESSATRE A UNE GERMIṄATION DANS GHAQUE INTERVALLE.

\begin{tabular}{|c|c|c|c|c|c|c|}
\hline \multirow[t]{3}{*}{ - } & \multicolumn{2}{|c|}{$\begin{array}{l}\text { Première } \\
\text { expérience }\end{array}$} & \multicolumn{2}{|c|}{$\begin{array}{l}\text { Deuxième } \\
\text { expérience }\end{array}$} & \multirow{3}{*}{$\begin{array}{c}\begin{array}{c}\text { Troisième } \\
\text { expérience }\end{array} \\
\text { Minutes }\end{array}$} & \multirow{3}{*}{$\begin{array}{l}\begin{array}{l}\text { Quatrième } \\
\text { expérience }\end{array} \\
\text { Minutes }\end{array}$} \\
\hline & (a) & $(b)$ & (a) & (b) & & \\
\hline & Minutes & Minutes & Minutes & Minutes & & \\
\hline Pendant les 4 premières heures .... & 31 & 33 & 30 & 31 & 31 & 34 \\
\hline De la $4^{\mathrm{e}}$ à la $6^{\mathrm{e}}$ heure $\ldots \ldots \ldots \ldots$ & 26 & 27 & 27 & 27 & 24 & 30 \\
\hline De la $6^{\text {e }}$ à la $7^{e}$ heure & & 31 & 41 & 37 & 50 & 40 \\
\hline De la $7^{e}$ à la $8^{\mathrm{e}}$ heure & 44 & 55 & 37 & 39 & 57 & 40 \\
\hline De la $8^{e}$ à la $9^{e}$ heure $\ldots \ldots \ldots \ldots$ & 55 & 73 & (?) & - & 一 & 38 \\
\hline
\end{tabular}

Ces chiffres, très approximatifs, que nous donnons à titre de simple curiosité, nous montrent que les générations de colibacilles se succèdent, à $37^{\circ}$ dans le lait, au rythme de 1 ou 2 à l'heure (30 à 60 minutes entre deux générations consécutives).

Ces durées semblent augmenter au fur et à mesure de l'incubation, ce qui paraît logique en raison de la concurrence vitale.

$2^{\circ}$ Le point capital pour nous, en ce moment, e'est le deuxième tableau, lequel représente le taux de multiplication à partir d'un germe, dans un temps donné. Nous avons été tentés de traduire ces résultats par des courbes qui seraient très évocatrices. Mais la multiplication des germes est tellement rapide qu'il faut renoncer à une telle entreprise, du moins sous cette forme. Aussi nous avons pensé, et nous proposons aux bactériologistes de faire comme nous, à exprimer, au lieu des nombres de germes, les logarithmes de ces nombres. Leur traduction sous forme de courbes devient alors possible, leurs variations s'étudient aisément et leur maniement est plus facile. C'est à un souci analogué, somme toute, que répond la notation $p \mathrm{H}$ imaginée par SöRENSEN pour représenter les concentrations en ions $\boldsymbol{H}$.

Nous proposons de la même façon, de représenter, quand ce sera nécessaire, le nombre de germes contenus dans $1 \mathrm{~cm}^{3}$ de milieu par le symbole Lg (ou logarithme du nombre de germes). Nous nous proposons d'ailleurs de reprendre cette idée à propos d'autres applications pratiques.

Ici, l'application de ce procédé commode et logique nous permet de construire des courbes fort intéressantes (p. 1069) correspondant au tableau suivant. (Pour rendre ce mode de représentation encore plus saisissant nous utiliserons les décalogarithmes). 


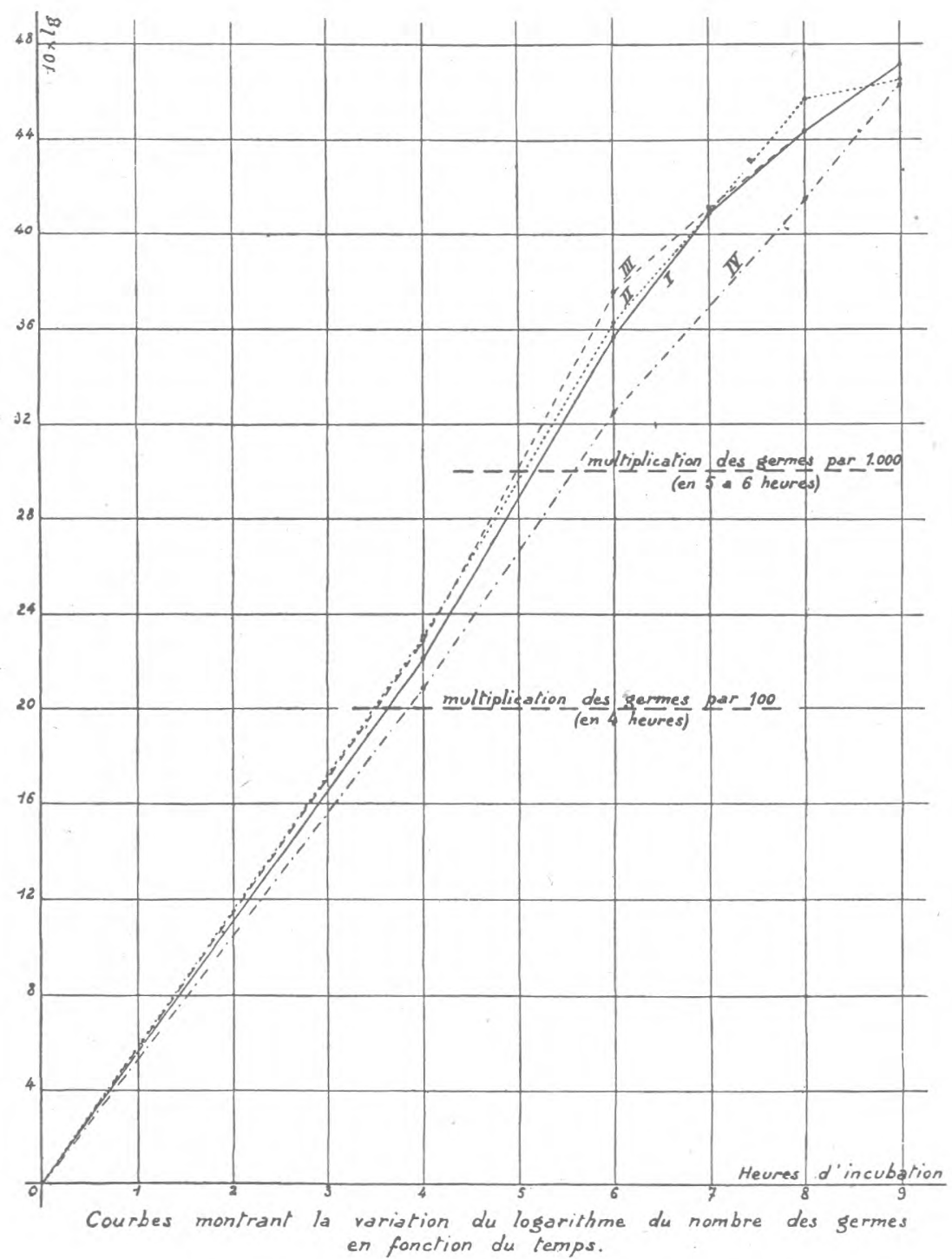

Ces nombres se prêtent aisément à une représentation graphique, de même que ceux qui expriment le $p H$ dans les travaux sur l'acidité potentielle, alors que ni les nombres de germes réels ni les concentrations en ions $H$ ne permettent de le faire. Sur le graphique, nous n'avons fait figurer que quatre courbes. Dans les deux premières expériences, une seule courbe moyenne représente les essais faits en double : $(a)$ et $(b)$. 
TABLEAU V.

DÉCALOGARTTHMES DES NOMBRES DE GERMES DE $B$, COLI OBTENUS A PARTIR D'UN SEUL GERME.

\begin{tabular}{|c|c|c|c|c|c|c|}
\hline \multirow[b]{3}{*}{ Au temps initial ............. } & \multicolumn{2}{|c|}{$\begin{array}{l}\text { Première } \\
\text { expérience }\end{array}$} & \multicolumn{2}{|c|}{$\begin{array}{l}\text { Deuxième } \\
\text { expérience }\end{array}$} & \multirow{3}{*}{$\begin{array}{c}\begin{array}{c}\text { Troisième } \\
\text { expérience }\end{array} \\
\mathrm{Lg}=0\end{array}$} & \multirow{3}{*}{$\begin{array}{r}\begin{array}{l}\text { Quatrième } \\
\text { expérience }\end{array} \\
\mathrm{Lg}=0\end{array}$} \\
\hline & (a) & $(b)$ & $(a)$ & $(b)$ & & \\
\hline & $\mathrm{Lg}=0$ & $\mathrm{Lg}=0$ & $\mathrm{Lg}=0$ & $\mathrm{Lg}=0$ & & \\
\hline Après 4 heures............... & 22,6 & 21,7 & 23,4 & 22,7 & 22,9 & 20,8 \\
\hline Après 6 heures............... & 36,3 & 35,1 & 36,7 & 35,9 & 37,6 & 32,5 \\
\hline Après 7 heures . . . . . . . . . . . . & - & 40,9 & 41,1 & 40,7 & 41,1 & 一 \\
\hline Après 8 heures . . . . . . . . . . . . & 44,5 & 44,1 & 46,0 & 45,4 & 44,3 & 41,5 \\
\hline Après 9 heures ............. & 47,8 & 46,6 & 46,5 & - & - & 46,3 \\
\hline
\end{tabular}

\section{CONCLUSIONS RELATIVES A LA PROLIFERATION DU $B$. COLI SEUL EN LAIT}

Le but poursuivi est le suivant : faire une incubation pendant un temps tel que dans le cas le plus défarorable, par exemple quelques germes dans un échantillon de $100 \mathrm{~cm}^{3}$ au temps initial, ceux-ci se soient suffisamment multipliés pour permettre un repiquage certain avec $1 \mathrm{~cm}^{3}$ ou même $1 / 10$ de centimètre cube.

Pour 1 germe dans $100 \mathrm{~cm}^{3}$ (soit $10 \mathrm{au}$ litre) il faut théoriquement une multiplication minimum par 100 si on repique $1 \mathrm{~cm}^{3}$. En pratique, à cause de l'hétérogénéité de la répartition des germes, il faut compter comme indispensable une multiplication par 500 ou même par 1.000. Autrement dit, il ne faudra pas faire de repiquages avant 5 à 6 heures d'incubation à $37^{\circ}$, si l'échantillon ne dépasse pas $100 \mathrm{~cm}^{3}$ (recherche limitée à 10 coli au litre - ce qui n'est pas trop de sévérité pour le contrôle d'un pasteurisateur qui n'en doit pas laisser $d u$ tout). Encore, le repiquage devra-t-il dans ces conditions, être fait sur $1 \mathrm{~cm}^{3}$ et non pas sur $1 / 10$ - ce qui exigerait un temps d'incubation supérieur.

Telles sont les conditions pratiques minima afférentes à une situation théorique où le coli est seul dans l'échantillon.

$$
\text { *** } *
$$

Cette condition minimum de 5 à 6 heures d'incubation pour la recherche du coli dans un échantillon de $100 \mathrm{~cm}^{3}$ de lait paraissant fixée, notre but est maintenant d'étudier ce qui se passera lorsque l'échantillon contiendra d'autres germes, et en particulier des ferments lactiques. La condition précédente, qui est nécessaire, va-t-elle être suffisante? Le développement des lactiques, leur présence, l'acidité formée, vont-ils gêner le développement du coli et rendre l'incubation impossible? 
La suite de notre étude se propose précisément de répondre à ces diverses questions, en vue d'arriver à fixer une méthode analytique sérieuse fondée sur des bases sûres.

\title{
BIBLIOGRAPHIE.
}

[1] M. H. Mac Crady. Tables for rapid interpretation of fermentation tube results. Publ. Health Journ. Toronto, 1918, IX, pp. 201 à 220. Cité dans : Karl J. Demeter. Bakteriologische und biologische Untersuchungsmethoden. Handbuch der Milchwirtschaft, 1930, p. 372.

[2] W. Dorner et P. Demont. Recherches sur le procédé Burri de numération bactérienne. Le Lait, décembre 1931, pp. 1015-1016.

(A suivre.)

\section{ÉTUDE DES CHAMPIGNONS TROUVÉS DANS LE LAIT LA CRËIME ET LE BEURRE}

\author{
par \\ M. GRIMES \\ Section de Bactériologie laitière \\ et \\ H. A. CUMMINS et V. C. E. KENNELLY \\ Sertion de Botanique \\ Université de Cork (Etat libre d'Irlande). \\ (Suite).
}

Mucor racemosus Fres. (fig. 7, Pl. 17).

Mycelium rampant et dense, couleur variant du jaune sale au blane ou gris. Sporanges terminaux, sur sporangiophores ramifiés ou non ramifiés, de dimensions variables, et jaunes brunâtres. Les sporangiophores sont de longueur variable. Les chlamydospores sont caractéristiques, et présentes à la fois dans le mycelium et dans les sporangiophores. Elles sont globulaires, ou en forme de casque et contiennent des spores brillantes. La columelle est en forme de massue. La capsule se brise quand on la monte dans l'acide lactique et le bleu coton pour l'examiner et laisse un col autour de la columelle.

Fait cailler le lait-tournesol d'une façon très épaisse en 48 heures, changeant le bleu en blane pur, avant traces de couleur rouge à la surface.

Cultures sur lait. - Lait caillé en 48 heures, avec décoloration du tournesol, excepté à la surface, suivi par solubilisation lente du caillé.

Tolérance à la chaleur. - Croissance à $70^{\circ} \mathrm{C}$. Pas de croissance à $75^{\circ} \mathrm{C}$.

Tolérance au sel. - Pas de croissance en solution de CzapeK contenant $15 \%$ de NaCl.

$p H$. - Sur solution de CzapeK, le $p H$ du milieu devint 6,8 .

Gélatine. - Liquéfaction en 10 jours, coloration verte sur la surface inférieure.

Production de diastase. - Faible.

Mucor corymbifer Cohn (Absidia Lichtheimi Ledner) (fig. 8, Pl. 17).

Isolé à trois occasions. Il a un mycelium gris blanchâtre tressé lâchement, se 